\title{
ATOMIC CARBON IN THE UPPER ATMOSPHERE OF TITAN
}

\author{
X. Zhang ${ }^{1}$, J. M. AJELlo ${ }^{2}$, AND Y. L. Yung ${ }^{1}$ \\ ${ }^{1}$ Division of Geological and Planetary Sciences, California Institute of Technology, Pasadena, CA, 91125, USA \\ 2 Jet Propulsion Laboratory, California Institute of Technology, Pasadena, CA 91109, USA \\ Received 2009 September 28; accepted 2009 November 25; published 2009 December 11
}

\begin{abstract}
The atomic carbon emission $C_{I}$ line feature at $1657 \AA\left({ }^{3} P_{J}^{0}{ }^{3} P_{J}\right)$ in the upper atmosphere of Titan is first identified from the airglow spectra obtained by the Cassini Ultra-violet Imaging Spectrograph. A one-dimensional photochemical model of Titan is used to study the photochemistry of atomic carbon on Titan. Reaction between $\mathrm{CH}$ and atomic hydrogen is the major source of atomic carbon, and reactions with hydrocarbons $\left(\mathrm{C}_{2} \mathrm{H}_{2}\right.$ and $\left.\mathrm{C}_{2} \mathrm{H}_{4}\right)$ are the most important loss processes. Resonance scattering of sunlight by atomic carbon is the dominant emission mechanism. The emission intensity calculations based on model results show good agreement with the observations.
\end{abstract}

Key words: planets and satellites: individual (Titan) - radiative transfer - scattering

Online-only material: color figures

\section{INTRODUCTION}

Titan's airglow spectra measured by the Ultra-violet Imaging Spectrograph (UVIS) on Cassini on 2004 December 13 provide the first detection of atomic carbon emission features in Titan's upper atmosphere (Ajello et al. 2008; Gustin et al. 2009). One of the strongest carbon $\mathrm{C}_{\mathrm{I}}$ line emissions is identified, corresponding to the resonance multiplets of neutral atomic carbon at $\left.1657 \AA{ }^{3} P_{J}^{0}-{ }^{3} P_{J}\right)$. The signal of the other $\mathrm{C}_{\mathrm{I}}$ line $\left(1561 \AA\left({ }^{3} D_{J}^{o}{ }^{3} P_{J}\right)\right)$ is below the noise level because its line intensity is weaker. This observation provides an opportunity to study atomic carbon in the upper atmosphere of Titan. In this Letter, first we use the Caltech/JPL one-dimensional photochemical model (Yung et al. 1984; Moses et al. 2000, 2005; Liang et al. 2007) to simulate the concentration and distribution of atomic carbon, and discuss its major sources and sinks. We then calculate the limb emission intensities based on the model results for the two C I lines and compare the $1657 \AA$ line intensities to the observation. This is followed by comparisons with previous studies of carbon atoms in the atmosphere of Mars and Venus (e.g., McElroy \& McConnell 1971; Fox 1982) and a discussion of the implications of the model on the chemistry for Titan.

\section{PHOTOCHEMICAL MODEL}

It is difficult for photons or electrons to dissociate $\mathrm{CH}_{4}$ and produce atomic carbon, because of the high binding energy of the $\mathrm{C}-\mathrm{H}$ bond in the $\mathrm{CH}_{4}$ molecule (Pang et al. 1987). Breaking all four $\mathrm{C}-\mathrm{H}$ bonds per mole of $\mathrm{CH}_{4}$ requires $1662 \mathrm{~kJ}$. Indeed, the main production mechanism of atomic carbon on Titan is via the production of the $\mathrm{CH}$ radical in a chain of chemical reactions initialized by the photolysis of $\mathrm{CH}_{4}$ by sunlight with wavelengths shorter than $1600 \AA$ (Yung et al. 1984). Figure 1 illustrates the important reaction pathways for producing and destroying atomic carbon and related hydrocarbons in the upper atmosphere of Titan.

The concentration profile of atomic carbon in the upper atmosphere of Titan is simulated by the Caltech/JPL kinetics one-dimensional photochemical model (Yung \& Allen 1984; Moses et al. 2000, 2005). In this model, we took into account species including the most abundant hydrocarbons and nitriles and solved the mass continuity equation above the tropopause $(\sim 50 \mathrm{~km})$ :

$$
\frac{\partial n_{i}}{\partial t}+\frac{\partial \varphi_{i}}{\partial z}=P_{i}-L_{i}
$$

where $n_{i}$ is the number density for species $i, \varphi_{i}$ is the vertical flux, $P_{i}$ is the chemical production rate, and $L_{i}$ is the chemical loss rate, all evaluated at time $t$ and altitude $z$. The vertical flux is given by

$$
\begin{aligned}
\varphi_{i}= & -\frac{\partial n_{i}}{\partial z}\left(D_{i}+K_{z z}\right)-n_{i}\left(\frac{D_{i}}{H_{i}}+\frac{K_{z z}}{H_{a}}\right)-n_{i} \frac{\partial T}{\partial z} \\
& \times\left[\frac{\left(1+\alpha_{i}\right) D_{i}+K_{z z}}{T}\right],
\end{aligned}
$$

where $K_{z z}$ is the eddy diffusion coefficient, $D_{i}$ is the molecular diffusion coefficient, $H_{i}$ is the scale height for species $i, H_{a}$ is the atmospheric scale height, $\alpha_{i}$ is the thermal diffusion parameter, and $T$ is the temperature.

The photochemical reactions are taken from the list in Moses et al. (2005). In Table 1, we summarize the important production and loss processes of atomic carbon and the corresponding integrated column reaction rates in our model. The reaction numbers are exactly the same as those in Moses et al. (2005) except for the $\mathrm{CH}$ photolysis reaction (R164). Therefore, all the reaction rate constants and related references (except R164) can be found in the auxiliary material Table S3 of that paper. The cross sections of $\mathrm{CH}$ photo-dissociation (R164) are obtained from van Dishoeck (1988).

The mixing ratio of $\mathrm{CH}_{4}$ in the deep atmosphere is prescribed to be $2.275 \times 10^{-2}$ (Liang et al. 2007), and we fixed the atomic hydrogen and molecular hydrogen escape velocities in the top atmosphere as $2.5 \times 10^{4} \mathrm{~cm} \mathrm{~s}^{-1}$ and $6.1 \times$ $10^{3} \mathrm{~cm} \mathrm{~s}^{-1}$, respectively. All species except hydrogen and carbon monoxide will leave the lower boundary with the velocity $2 \times 10^{-4} \mathrm{~cm} \mathrm{~s}^{-1}$, due to the cold trap at the tropopause. The atmospheric temperature profile is based on the Cassini measurements (Liang et al. 2007). The eddy diffusion coefficient profile is constrained by recent Cassini UVIS stellar occultation data (D. E. Shemansky 2009, private communication). Above the homopause (around $1000 \mathrm{~km}$ ), the molecular diffusion process dominates. 


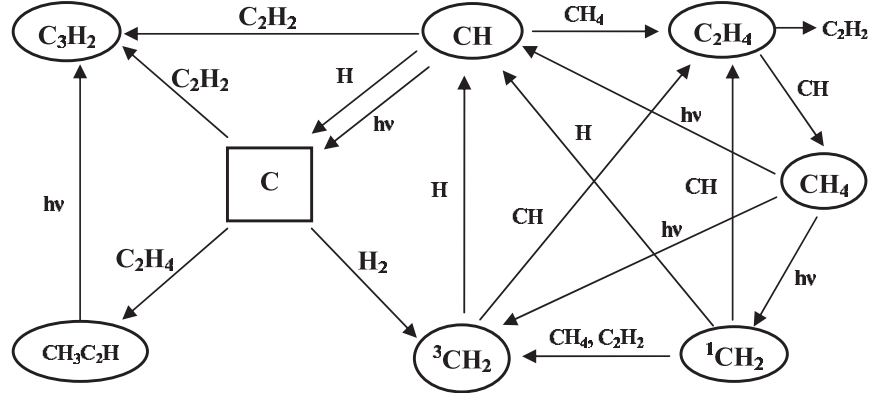

Figure 1. Schematic diagram illustrating the important reaction pathways related to atomic carbon.

Table 1

Important Reactions Involving Atomic Carbon

\begin{tabular}{ll}
\hline \hline \multicolumn{1}{c}{ Reaction } & Integrate Column Reaction Rate \\
\hline Sources & \\
$(\mathrm{R} 182) \mathrm{H}+\mathrm{CH} \rightarrow \mathrm{C}+\mathrm{H}_{2}$ & $2.014 \times 10^{7}$ \\
$(\mathrm{R} 164) \mathrm{CH} \rightarrow \mathrm{C}+\mathrm{H}$ & $1.078 \times 10^{5}$ \\
Sinks & $1.003 \times 10^{7}$ \\
$(\mathrm{R} 238) \mathrm{C}+\mathrm{H}_{2} \stackrel{\mathrm{M}}{\rightarrow}{ }^{3} \mathrm{CH}_{2}$ & $9.634 \times 10^{6}$ \\
$(\mathrm{R} 239) \mathrm{C}+\mathrm{C}_{2} \mathrm{H}_{2} \stackrel{\mathrm{M}}{\rightarrow} \mathrm{C}_{3} \mathrm{H}_{2}$ & $5.819 \times 10^{5}$ \\
$(\mathrm{R} 240) \mathrm{C}+\mathrm{C}_{2} \mathrm{H}_{4} \stackrel{\mathrm{M}}{\rightarrow} \mathrm{CH}_{3} \mathrm{C}_{2} \mathrm{H}$ & \\
\hline
\end{tabular}

Notes. The reaction numbers are the same as those in Moses et al. (2005) except the R164. All the reaction rate constants and related references (except R164) can be found in the auxiliary material Table S3 of that paper. The cross sections of $\mathrm{CH}$ photodissociation (R164) are obtained from van Dishoeck (1988).

${ }^{\text {a }}$ Integrated column reaction rate is in units of $\mathrm{cm}^{-2} \mathrm{~s}^{-1}$.

More than $80 \%$ of $\mathrm{CH}$ radicals are removed by reacting with $\mathrm{CH}_{4}$ to form $\mathrm{C}_{2} \mathrm{H}_{4}$, but a small portion of $\mathrm{CH}$ (less than $1 \%$ in the upper atmosphere) react with atomic hydrogen to produce carbon atoms (R182 in Table 1):

$$
\mathrm{H}+\mathrm{CH} \rightarrow \mathrm{C}+\mathrm{H}_{2} \text {. }
$$

Atomic carbon can also be produced by the $\mathrm{CH}$ photodissociation (R164), but the production rate is negligible compared with the R182 (see the discussion below). The major loss of atomic carbon is through the reaction with molecular hydrogen to form $\mathrm{CH}_{2}$ (R238) in the lower atmosphere. In the upper atmosphere, the dominant sinks of atomic carbon are the reactions with hydrocarbons like $\mathrm{C}_{2} \mathrm{H}_{2}(\mathrm{R} 239)$ and $\mathrm{C}_{2} \mathrm{H}_{4}(\mathrm{R} 240)$ to produce $\mathrm{C}_{3}$ hydrocarbons.

The concentration profiles of atomic carbon together with $\mathrm{CH}, \mathrm{CH}_{4}$, and atomic hydrogen above $400 \mathrm{~km}$ are shown in the left panel of Figure 2. Two $\mathrm{CH}_{4}$ observation data points from Niemann et al. (2005) and Waite et al. (2005) are also shown in the figure. The plot shows that our model results match the measurements very well. The $\mathrm{CH}$ abundance is roughly 100 molecules $\mathrm{cm}^{-3}$ at its peak around $1250 \mathrm{~km}$. Our model results are in general agreement with recent models of Titan (Dobrijevic et al. 2008; Krasnopolsky 2009). The similar pattern of the $\mathrm{H}$ and $\mathrm{C}$ concentration profiles suggests that the production of $\mathrm{C}$ is directly correlated with abundance of $\mathrm{H}$ (R182), not only because $\mathrm{H}$ is one of the reactants, but also because the atomic hydrogen is directly involved in the production processes of $\mathrm{CH}$ (Figure 1).

The right panel of Figure 2 shows the production and loss rates involved in the photochemistry of atomic carbon. The production rate of R164 is two orders of magnitude less than
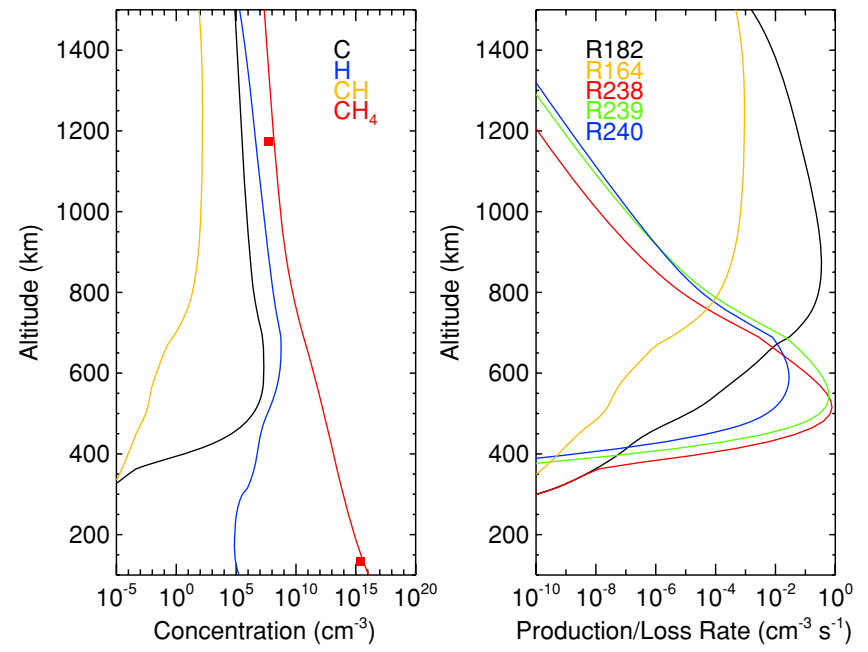

Figure 2. Left panel: concentration profiles of atomic carbon, $\mathrm{CH}, \mathrm{CH}_{4}$ ' and atomic hydrogen (labeled by colors). Two $\mathrm{CH}_{4}$ observation data points from Niemann et al. (2005) and Waite et al. (2005) are shown by filled squares. Right panel: production rate (R182 and R164) and loss rate (R238, R239, and R240) of atomic carbon due to the most important four reactions (labeled by colors). (A color version of this figure is available in the online journal.)

that of $\mathrm{R} 182$ at $1200 \mathrm{~km}$. Therefore, the $\mathrm{CH}+\mathrm{H}$ reaction $(\mathrm{R} 182)$ is the dominant source of atomic carbon. The loss rate curves display clearly that in the lower atmosphere of Titan, the reaction with $\mathrm{H}_{2}$ is the major loss process of $\mathrm{C}(\mathrm{R} 238)$. However, in the upper atmosphere (above $800 \mathrm{~km}$ ), $\mathrm{C}_{2} \mathrm{H}_{2}$ and $\mathrm{C}_{2} \mathrm{H}_{4}$ dominate the loss of C (R239 and R240). At $1200 \mathrm{~km}$, the contribution of R239 to the loss rate is about $34 \%$ and that of R240 is about $61 \%$.

The contribution of nitrogen chemistry to the $\mathrm{C}$ production is negligible. The main sources of $\mathrm{C}$ in nitrogen chemistry are the photolysis of $\mathrm{CN}$ and the reaction between $\mathrm{N}$ atom and $\mathrm{CN}$, which is analogous to R182:

$$
\mathrm{N}+\mathrm{CN} \rightarrow \mathrm{C}+\mathrm{N}_{2} .
$$

In our model, the abundances of $\mathrm{CN}$ and $\mathrm{N}$ are about $1 \times$ $10^{3} \mathrm{~cm}^{-3}$ and $1 \times 10^{5} \mathrm{~cm}^{-3}$ in the upper atmosphere. The photolysis rate of $\mathrm{CN}$ above $1000 \mathrm{~km}$ is around $3 \times 10^{-8} \mathrm{~s}^{-1}$. Therefore, the $C$ production rate is on the order of $10^{-5} \mathrm{~cm}^{-3} \mathrm{~s}^{-1}$ due to $\mathrm{CN}$ photodissociation. The rate coefficient for the reaction between N and CN, according to Atakan \& Wolfrum (1992), is $3.24 \times 10^{-13} \mathrm{e}^{(-1771 / T)}$ between $298 \mathrm{~K}$ and $534 \mathrm{~K}$. In the upper atmosphere of Titan, the rate should be even smaller because the temperature is cooler than $150 \mathrm{~K}$. Actually, the production rate of $\mathrm{C}$ due to this reaction is around $10^{-10} \mathrm{~cm}^{-3} \mathrm{~s}^{-1}$ above $1000 \mathrm{~km}$. Both of these two production rates are negligible compared with the production rate from R182, which is on the order of $10^{-2} \mathrm{~cm}^{-3} \mathrm{~s}^{-1}$ based on the reaction rate coefficient from van Harrevelt et al. (2002).

Given the main sources and sinks of atomic carbon, the steady-state concentration of which can be expressed as (the diffusion term is ignored because the chemical reaction timescale is short)

$$
\frac{[\mathrm{C}]}{[\mathrm{CH}]}=\frac{J_{164}+k_{182}[\mathrm{H}]}{k_{238}[\mathrm{H}][\mathrm{M}]+k_{239}\left[\mathrm{C}_{2} \mathrm{H}_{2}\right][\mathrm{M}]+k_{240}\left[\mathrm{C}_{2} \mathrm{H}_{4}\right][\mathrm{M}]},
$$

where $\mathrm{M}$ is the bath gas and $J_{164}$ refers to the photolysis rate coefficient. $k_{182}, k_{238}, k_{239}$, and $k_{240}$ are the reaction rate coefficients for R182, R238, R239, and R240, respectively. The 
Table 2

Atomic Data for the Transitions of the Mulitplets 2 and 3 in C I

\begin{tabular}{lcc}
\hline \hline \multicolumn{1}{c}{ Transition } & $\lambda(\AA)$ & $f\left(10^{-2}\right)$ \\
\hline $2 p^{2} 3 P-3 s{ }^{3} P^{\circ}$ & & \\
${ }^{3} P_{1}-{ }^{3} P_{2}$ & 1656.2672 & 5.89 \\
${ }^{3} P_{0}-{ }^{3} P_{1}$ & 1656.9283 & 13.9 \\
${ }^{3} P_{2}-{ }^{3} P_{2}$ & 1657.0082 & 10.4 \\
${ }^{3} P_{1}-{ }^{3} P_{1}$ & 1657.3792 & 3.56 \\
${ }^{3} P_{1}-{ }^{3} P_{0}$ & 1657.9068 & 4.73 \\
${ }^{3} P_{2}-{ }^{3} P_{1}$ & 1658.1212 & 3.56 \\
$2 p^{2} 3 P-2 p$ & \\
${ }^{3} P_{0}-{ }^{3} D_{1}$ & & 7.19 \\
${ }^{3} P_{1}-{ }^{3} D_{2}$ & 1560.3092 & 5.39 \\
${ }^{3} P_{1}-{ }^{3} D_{1}$ & 1560.6822 & 1.80 \\
${ }^{3} P_{2}-{ }^{3} D_{2}$ & 1560.7090 & 1.08 \\
${ }^{3} P_{2}-{ }^{3} D_{1}$ & 1561.3402 & 0.07 \\
${ }^{3} P_{2}-{ }^{3} D_{3}$ & 1561.3667 & 6.03 \\
\hline
\end{tabular}

concentration of $\mathrm{CH}$ is nearly independent of $\mathrm{C}$ because most of the $\mathrm{CH}$ radicals are destroyed by reacting with $\mathrm{CH}_{4}$ in the high altitude. A full set of photochemical reactions (more than 900 reactions) is used to model the concentrations of all the hydrocarbon species and gives robust results for $\mathrm{C}_{2} \mathrm{H}_{2}, \mathrm{C}_{2} \mathrm{H}_{4}$, $\mathrm{CH}$, and $\mathrm{H}$. The main uncertainty is due to the reaction R182. From Equation (3), because $J_{164}$ can be neglected compared with the reaction rate of $\mathrm{CH}+\mathrm{H}$, the concentration of atomic carbon is proportional to the rate coefficient $k_{182}$. For example, in our study, we use the reaction rate coefficient from van Harrevelt et al. (2002) and the atomic carbon density is $2.7 \times 10^{7} \mathrm{~cm}^{-3}$ at $1200 \mathrm{~km}$. This value is $30 \%$ larger than the carbon abundance $\left(2.0 \times 10^{7} \mathrm{~cm}^{-3)}\right.$ calculated based on the rate coefficient from Harding et al. (1993). The ratio of the two rate coefficients under the temperature at $1200 \mathrm{~km}$ is roughly 1.3 . However, our radiative modeling study (Section 3) shows that the limb emission spectrum is not very sensitive to the $30 \%$ concentration difference.

\section{CARBON EMISSIONS}

Previous studies suggest that the resonance scattering of sunlight by atomic carbon contributes the most to the observed intensities at $1561 \AA$ and $1657 \AA$ (e.g., McElroy \& McConnell 1971). In order to compare our photochemical model results with observed airglow data, we computed the resonant scattering emission intensities of the carbon $C_{\mathrm{I}}$ lines at $1561 \AA$ and $1657 \AA$ by a pseudo-spherical radiative transfer method. First we assume a local plane parallel layering atmosphere. Based on the onedimensional photochemical model results, the source function for each layer is computed from a multiple scattering calculation by the DISORT model (Stamnes et al. 1988). The limb radiance can be obtained by solving the radiative transfer equation along the line of sight.

For each transition line, the absorption coefficient per atom at rest can be expressed as $\frac{\pi e^{2}}{m c} f$, where $f$ is the oscillator strength and $\pi e^{2} / m c\left(=2.647 \times 10^{-2} \mathrm{~cm}^{2} \mathrm{~s}^{-1}\right)$ is the integrated absorption coefficient per atom for unit $f$-value, and $e$ and $m$ are the charge and mass of an electron, respectively. The atomic data for the transitions of the mulitplets 2 and 3 in $C_{\text {I }}$ are shown in Table 2. The $f$-values and vacuum wavelengths are obtained from Wiese et al. (1996) and Morton (1991), respectively. When the carbon atoms are in thermal motion, the absorption line shape follows a Voigt profile, which is the convolution of the Lorentz and Doppler (Gaussian) line shapes. The pressure
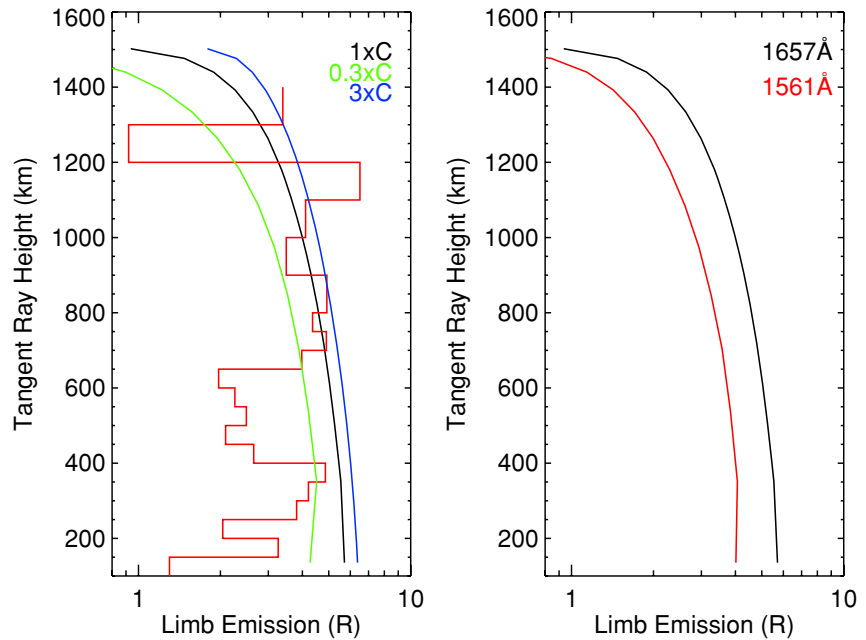

Figure 3. Left panel: modeled limb intensities for $1657 \AA$, compared with Cassini limb observational data (red line). The intensities are in units of Rayleigh (1 Rayleigh $(\mathrm{R})=10^{6}$ photons $\mathrm{cm}^{-2} \mathrm{~s}^{-1}$ ). A sensitivity study has been made for 0.3 times and 3 times the carbon abundances from the photochemical model results. Right panel: modeled limb intensities for $1657 \AA$ (black line) and $1561 \AA$ (red line).

(A color version of this figure is available in the online journal.)

broadening effect is negligible in the upper atmosphere of Titan. Therefore, the Doppler line profile is dominant, and the FWHM of each line is roughly proportional to the square root of the atmospheric temperature, on the order of several mA. The single scattering albedo of atomic carbon is approximately unity because in the upper atmosphere of Titan, the optical depth of atomic carbon is about $1-10$ (at the line center), whereas the optical depth from all of the other gas species is only on the order of $10^{-3}$ and the contribution from the tholin is roughly $10^{-2}$ based on the tholin profile from Liang et al. (2007). We took solar C I emission spectra on 2004 December 13 from the Composite Solar Ultraviolet Spectral Irradiance Data Set (Deland \& Cebula 2008), which provide the daily average solar spectra over the wavelength range $120-400 \mathrm{~nm}$ in $1 \mathrm{~nm}$ bins from 1978 November 8 to 2005 August 1.

The limb intensities of resonance scattering emissions are shown in Figure 3. The left panel shows the modeled limb emissions at $1657 \AA$ for each tangent ray height and compared with the observed data (in red). The measured intensities are determined by differencing in 50 and $100 \mathrm{~km}$ increments the intensities of the UVIS limb airglow spectral feature at $1657 \AA$ (within the $5.5 \AA$ A FWHM bandpass) with the composite airglow model: a sum of (1) reflected sunlight from Rayleigh scattering and (2) Lyman-Birge-Hopfield (LBH) band system and N I multiplet airglow emissions (Ajello et al. 2008).

Our photochemical model results agree with the Cassini data above $600 \mathrm{~km}$. The left panel of Figure 3 also illustrates the sensitivity of the carbon abundances to the modeled emission intensities. Since the upper atmosphere is optically thick due to strong carbon absorption, the change of carbon number density by a factor of 3 results in a change of emission intensity by only $20 \%-50 \%$, approximately. We attribute the discrepancy between the model and data to the error associated in subtracting the large contribution of reflected sunlight at $1657 \AA$ (Ajello et al. 2008). The predicted limb emission of $1561 \AA$ is shown in the right panel. The limb emission profiles for the two lines are very similar. The magnitude at $1561 \AA$ is only $30 \%$ of that at $1657 \AA$ although the total line intensity at $1561 \AA$ is about half 
of that at $1657 \AA$. The reason is that the emission efficiency is reduced by the optically thick upper atmosphere.

\section{DISCUSSION AND CONCLUSIONS}

The carbon atoms have been observed on Mars and Venus. The photochemistry of atomic carbon in the upper atmosphere of Mars was first studied by McElroy \& McConnell (1971) based on the Mars-Mariner emission spectra (Barth et al. 1971). They also discussed the abundance and profile of atomic carbon in the upper atmosphere of Venus, but no spectral data were available until the Pioneer Venus Orbiter measurements (Stewart et al. 1979; Niemann et al. 1980; Taylor et al. 1980). Based on these data, Fox (1982) and Paxton (1985) used photochemical models to investigate the reactions of atomic carbon and possible emission mechanisms on Venus. Both of the two previous studies show that the production mechanisms of atomic carbon on Mars and Venus are photodissociation and electron-impact dissociation of $\mathrm{CO}_{2}$ and $\mathrm{CO}$, and the primary sink of $\mathrm{C}$ is the reaction with $\mathrm{O}_{2}$, along with some contribution from the reaction with NO. Fox (2004) and Fox \& Paxton (2005) revisited the photochemistry of atomic carbon for Mars and Venus, respectively. Fox (2004) discovered that dissociative recombination $\mathrm{CO}_{2}^{+}$is the most important source of atomic carbon in the Martian thermosphere. Fox \& Paxton (2005) confirmed that the CO photodissociation is the dominant production process of atomic carbon on Venus.

By contrast, in a non-oxidizing environment such as Titan, the photochemistry of atomic carbon is quite different. As a natural laboratory of hydrocarbon chemistry, Titan starts its photochemistry from the photolysis of $\mathrm{CH}_{4}$. Atomic carbon only plays a minor role in one small branch of the complex network of all known hydrocarbon reactions (Figure 9 in Moses et al. 2005). Our study suggests that the nitrogen chemistry contributes little to the production of $\mathrm{C}$. Atomic hydrogen is a key reactant in producing atomic carbon, and the $\mathrm{C}_{2}$ hydrocarbons like $\mathrm{C}_{2} \mathrm{H}_{2}$ and $\mathrm{C}_{2} \mathrm{H}_{4}$ above $600 \mathrm{~km}$ dominate the loss processes. Compared with Venus and Mars, the photochemistry of $\mathrm{C}$ in Titan's upper atmosphere is relatively simple because there is no direct source from photon- or electron-induced dissociation. However, since $\mathrm{C}$ is related to several of the most important species, for example, $\mathrm{CH}_{4}, \mathrm{CH}, \mathrm{H}, \mathrm{C}_{2} \mathrm{H}_{2}$, and $\mathrm{C}_{2} \mathrm{H}_{4}$, a full set of photochemical reactions (at least the $\mathrm{C}_{3}$ chemistry) needs to be taken into account. Based on the current knowledge of photochemistry and transport processes on Titan, our onedimensional photochemical model is able to produce an atomic carbon vertical profile, based on which we can reproduce the Cassini limb observation at $1657 \AA$ Ay simple resonant scattering calculation. This agreement confirms previous results in other planets that the observed intensities at $1561 \AA$ and $1657 \AA$ arise mostly from the resonance scattering of sunlight by atomic carbon, and shows that our knowledge of the photochemical behavior of C in Titan's upper atmosphere is roughly complete. We also predict the $1561 \AA$ limb emission intensity, which remains to be confirmed by future observations.

We thank M. C. Liang and J. I. Moses for making their updated kinetics for the Titan model available and D. E. Shemansky for providing the Cassini UVIS stellar occultation data, V. Natraj, M. Line, and M. Gerstell for reading the manuscript. We thank an anonymous referee for providing updates of reaction coefficients. The research was supported in part by NASA PATM grant NNX09AB72G to the California Institute of Technology.

\section{REFERENCES}

Ajello, J. M., et al. 2008, Geophys. Res. Lett., 35, L06102

Atakan, B., \& Wolfrum, J. 1992, Chem. Phys. Lett., 186, 547

Barth, C. A., Hord, C. W., Pearce, J. B., Kelly, K. K., Anderson, G. P., \& Stewart, A. I. 1971, J. Geophys. Res., 76, 2213

Deland, M. T., \& Cebula, R. P. 2008, J. Geophys. Res., 113, A11103

Dobrijevic, M., Carrasco, N., Hébrard, E., \& Pernot, P. 2008, Planet. Space Sci., 56,1630

Fox, J. L. 1982, J. Geophys. Res., 87, 9211

Fox, J. L. 2004, J. Geophys. Res., 109, A08306

Fox, J. L., \& Paxton, L. J. 2005, J. Geophys. Res., 110, A01311

Gustin, J., Ajello, J. M., Stevens, M. H., Stepfan, A. W., Stewart, I., Larsen, K., Esposito, L., \& McClintock, W. 2009, Geophys. Res. Lett.,, submitted

Harding, L. B., Guadagnini, R., \& Schatz, G. C. 1993, J. Phys. Chem., 97, 5472

Krasnopolsky, V. A. 2009, Icarus, 201, 226

Liang, M., Yung, Y. L., \& Shemansky, D. E. 2007, ApJ, 661, L199

McElroy, M. B., \& McConnell, J. C. 1971, J. Geophys. Res., 76, 6674

Morton, D. C. 1991, ApJS, 77, 119

Moses, J. I., Bezard, B., Lellouch, E., Gladstone, G. R., Feuchtgruber, H., \& Allen, M. 2000, Icarus, 143, 244

Moses, J. I., Fouchet, T., Bézard, B., Gladstone, G. R., Lellouch, E., \& Feuchtgruber, H. 2005, J. Geophys. Res., 110, E08001

Niemann, H. B., Kasprzak, W. T., Hedin, A. E., Hunten, D. M., \& Spencer, N. W. 1980, J. Geophys. Res., 85, 7817

Niemann, H. B., et al. 2005, Nature, 438, 779

Pang, K. D., Ajello, J. M., Franklin, B., \& Shemansky, D. E. 1987, J. Chem. Phys., 86, 2750

Paxton, L. J. 1985, J. Geophys. Res., 90, 5089

Stamnes, K., Tsay, S.-C., Jayaweera, K., \& Wiscombe, W. 1988, Appl. Opt., 27,2502

Stewart, A. I., Anderson, D. E., Esposito, L. W., \& Barth, C. A. 1979, Science, 203, 777

Taylor, H. A., Brinton, H. C., Bauer, S. J., Hartle, R. E., Cloutier, P. A., \& Daniell, R. E. 1980, J. Geophys. Res., 85, 7765

van Dishoeck, E. F. 1988, in Rate Coefficients in Astrochemistry, ed. T. J. Millar \& D. A. Williams (Dordrecht: Kluwer), 49

van Harrevelt, R., van Hemert, M. C., \& Schatz, G. C. 2002, J. Chem. Phys., 116,6002

Waite, J. H., Jr., et al. 2005, Science, 308, 982

Wiese, W. L., Fuhr, J. R., \& Deters, T. M. (ed.) 1996, in J. Phys. Chem. Ref. Data, Mono., Vol. 7, Atomic Transition Probabilities of Carbon, Nitrogen, and Oxygen: A Critical Data Compilation (Washington, DC: American Chemical Society)

Yung, Y. L., Allen, M., \& Pinto, J. P. 1984, ApJS, 55, 465 